\title{
S? \\ Universal Optimal Transmission of Light Through Disordered Materials
}

\author{
I. M. Vellekoop and A. P. Mosk \\ Complex Photonic Systems, Faculty of Science and Technology, and MESA ${ }^{+}$Institute for Nanotechnology, University of Twente, \\ P.O. Box 217, 7500 AE Enschede, The Netherlands
}

(Received 3 April 2008; revised manuscript received 26 June 2008; published 15 September 2008)

\begin{abstract}
We experimentally demonstrate increased diffuse transmission of light through strongly scattering materials. Wave front shaping is used to selectively couple light to the open transport eigenchannels, specific solutions of Maxwell's equations which the sample transmits fully, resulting in an increase of up to $44 \%$ in the total angle-integrated transmission compared to the case where plane waves are incident. The results for each of several hundreds of experimental runs are in excellent quantitative agreement with random matrix theory. From our measurements we conclude that with perfectly shaped wave fronts the transmission of a disordered sample tends to a universal value of $2 / 3$, regardless of the thickness.
\end{abstract}

DOI: 10.1103/PhysRevLett.101.120601

PACS numbers: 05.60.- $\mathrm{k}$, 42.25.Dd, 73.23. $-\mathrm{b}$

The transport of waves in strongly scattering media is, usually, well described by diffusion theory. However, the diffusion equation does not take into account interference. Interference gives rise to fundamental effects such as enhanced backscattering [1,2] and Anderson localization of light [3-5]. Such interference effects may be observed with incident light in a single freely propagating mode (scattering channel), such as a plane incident wave. In 1984, Dorokhov predicted a striking multichannel interference effect. Using random matrix theory (RMT), he showed that the transmission through a diffusive material is the result of a small number of open eigenchannels with a transmission coefficient of close to one [6]. Each eigenchannel [7] corresponds to a specific linear combination of multiple free modes. Waves coupled to an open eigenchannel will be fully transmitted through a disordered sample, even if the sample is optically thick. In contrast, an incident plane wave couples mainly to closed eigenchannels (with a transmission coefficient of close to zero) and, consequently, most of its power is diffusely reflected. This result was originally obtained for electron transport in a wire. Later, it was generalized to a slab geometry [8] and to optical systems $[9,10]$.

Universal conductance fluctuations, which are random fluctuations in the coupling of waves to open and closed eigenchannels, have been observed in experiments [1114]; however, no controlled coupling to eigenchannels has been reported so far. Here we experimentally demonstrate the injection of light into the open eigenchannels of a strongly disordered sample, resulting in a large increase of the diffusely transmitted intensity. We accomplish this by shaping the wave front of the incident light, which amounts to individually controlling the phases of multiple incoming free modes.

The transmission amplitudes of waves through a mesoscopic medium are described by a transmission matrix $t$. The field in the outgoing free modes at the back of the sample is given by

$$
E_{b}=\sum_{a}^{N} t_{b a} E_{a},
$$

where indices $a$ and $b$ label incident and transmitted free modes, respectively. $N$ is the total number of incident free modes. Any complete orthogonal set of modes may be chosen; in mesoscopic physics it is usual to choose the transverse modes of a perfect waveguide as a basis, one may also work with an overcomplete basis of incident angles or diffraction limited spots on the sample surface. Transmission eigenchannels are defined for each individual sample by decomposing the transmission matrix as $t=$ $U \mathcal{T} V^{T}$. Here $U$ and $V$ are unitary matrices that perform the basis transformations between free modes outside the sample and eigenchannels inside. $\mathcal{T}$ is a real diagonal matrix containing eigenchannel transmission coefficients.

We use a spatial light modulator (see Fig. 1) to shape the wave front of the incident light. The computer program that controls the wave front shaper optimizes the intensity of a small target spot at the back surface of the sample using the measured intensity in the target as feedback [15]. This wave front shaping method creates a high intensity focus through the sample, which by itself is not a result of the bimodal distribution of the eigenchannel transmission coefficients. Importantly, the disordered background speckle around the focus turns out to be a very sensitive probe of the distribution of the elements of $\mathcal{T}$.

For example, for the limiting distribution where all eigenchannels are completely open $\mathcal{T}=0$ or completely closed $\mathcal{T}=1$ (the so-called maximal fluctuations [9]), only open eigenchannels will contribute to the intensity in the target. Therefore, the optimization algorithm will select only linear combinations corresponding to open eigenchannels. As a result, the diffuse transmission will increase so that the ideally shaped wave front will have a total transmission of unity. The distribution predicted by Dorokhov [6] is close to this limiting case. 
We now proceed to introduce a quantitative measure of the control we exert over a shaped wave front. Our algorithm maximizes the intensity in a diffraction limited spot, which is exactly one of the transmitted free modes. We label this special target mode with the index $\beta$. The ideally shaped incident wave front $E_{a}^{\mathrm{opt}, \beta}$ for maximizing the intensity in $\beta$ is given by [16]

$$
E_{a}^{\mathrm{opt}, \beta}=\left(T_{\beta}\right)^{-1 / 2} t_{\beta a}^{*}, \quad T_{\beta} \equiv \sum_{a}^{N}\left|t_{\beta a}\right|^{2},
$$

where $T_{\beta}$ normalizes the total incident power. Our optimization algorithm proceeds as follows: the matrix elements $t_{\beta a}$ are measured up to a constant prefactor by cycling the phase of the light in the incident mode $a$ while observing the intensity in target mode $\beta$ [15]. After $N$ phases have been measured, the optimized incident wave front is constructed according to Eq. (2). This optimized wave front couples to a superposition of eigenchannels, mostly to channels with high transmission eigenvalues.

In any experiment, the resolution and the spatial extent of the generated field are finite. Therefore, it will never be possible to exactly construct the wave front described by Eq. (2). To quantify how well the actual incident field $E_{a}^{\text {act, } \beta}$ matches the optimal incident field $E_{a}^{\mathrm{opt}, \beta}$, we introduce the overlap coefficient $\gamma$ as

$$
\gamma \equiv \sum_{a}^{N}\left(E_{a}^{\mathrm{opt}, \beta}\right)^{*} E_{a}^{\mathrm{act}, \beta} .
$$

The degree of intensity control is $|\gamma|^{2}$. We can now write any incident wave front as a linear superposition of the perfect wave front and an error term

$$
E_{a}^{\mathrm{act}, \beta}=\gamma E_{a}^{\mathrm{opt}, \beta}+\sqrt{1-|\gamma|^{2}} \Delta E_{a}
$$

where the error term $\Delta E_{a}$ is normalized. For ideal control over the incident wave front $|\gamma|^{2}=1$. In earlier experiments [15] the degree of control was relatively low $\left(|\gamma|^{2} \ll 0.1\right)$, and total transmission did not increase measurably. In this Letter, we discuss experiments at much higher values of $|\gamma|^{2}$, up to 0.33 .

The experimental apparatus (see Fig. 1) is designed to approach the optimal wave front as closely as possible, by controlling the largest possible fraction of the incident free modes. An expanded beam from a $632.8 \mathrm{~nm}$ HeNe laser is rotated to a $45^{\circ}$ linear polarization by a half wave plate and impinges on a polarizing beam splitter cube. Horizontally and vertically polarized beams are modulated with separate reflective liquid crystal displays (Holoeye LC-R 2500) and then recombined, to provide control over modes with both polarizations. We used a 4-pixel macropixel modulation method [17] to control the phase of the light without residual amplitude modulation. The modulator is divided into 3816 independently programmable segments. A computer programs the modulators using feedback from a

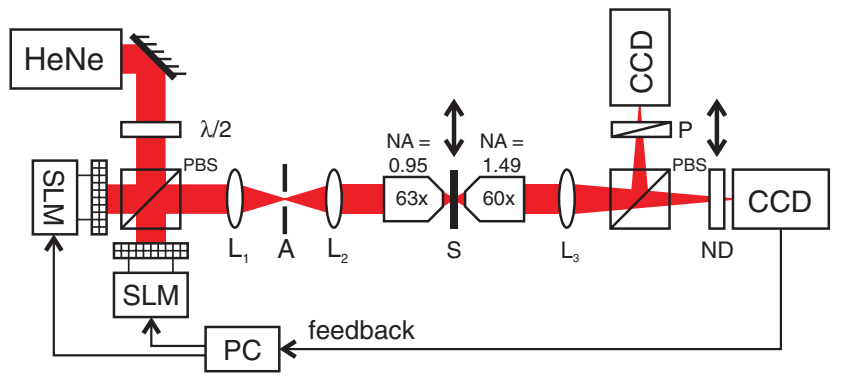

FIG. 1 (color). Experimental setup. HeNe, expanded $632.8 \mathrm{~nm}$ HeNe laser; $\lambda / 2$, half wave plate; PBS, polarizing beam splitter cube; SLM, spatial light modulator. A, iris diaphragm; 63×, microscope objective; $60 \times$, oil-immersion microscope objective; S, sample; P, polarizer; ND, neutral density filter; $\mathrm{L}_{1}, \mathrm{~L}_{2}$, $\mathrm{L}_{3}$, lenses with focal length of, respectively, 250, 200, and $600 \mathrm{~mm}$. ND and S are translated by computer controlled stages.

camera as discussed below. A sequential optimization algorithm [15] was used to optimize the wave front [16]. A high numerical aperture objective $(\mathrm{NA}=0.95$, Zeiss Achroplan $63 \times$ ) projects the shaped wave front onto the sample.

Each sample consists of a layer of spray-painted $\mathrm{ZnO}$ particles on a standard glass microscope cover slip. The particles have an average diameter of $200 \mathrm{~nm}$, which makes them strongly scattering for visible light. The mean free path was determined by measuring the total transmission and equals $0.85 \pm 0.15 \mu \mathrm{m}$ at a wavelength of $632.8 \mathrm{~nm}$. We used samples with thicknesses of 5.7 and $11.3 \mu \mathrm{m}$. The samples were positioned in the focal plane of the microscope objective to minimize the size of the diffuse spot, and thereby the number of contributing modes. The number of such contributing free modes was estimated from the intensity profile of the transmitted light [12] to be $5.5 \times 10^{3}$ and $1.0 \times 10^{4}$ modes, for the thin and the thick samples, respectively. The samples were mounted on a motorized stage to translate them in the focal plane.

A high NA oil-immersion objective (Nikon TIRF $60 \times /$ $\mathrm{NA}=1.49$ ) collects the transmitted light. The transmitted light is split into horizontal and vertical polarizations by a beam splitter cube. A second polarizer improves the extinction ratio for reflected light. The magnification of the detection system is $180 \times$, enough to well-resolve individual speckles. A camera measures the power of the horizontally polarized light in a disk with a diameter of $0.11 \mu \mathrm{m}$ at the sample, which is smaller than a single speckle, to provide feedback for the optimization algorithm.

After optimization, a calibrated neutral density filter with a transmission of $1.4 \times 10^{-3}$ is placed in front of the camera to measure the high intensity in the target. A second camera images the intensity of the vertically polarized light.

Optimizing the incident wave front caused the intensity in the target to increase dramatically. In Fig. 2 we plot the 
transmitted intensity through a $11.3 \mu \mathrm{m}$-thick sample for a nonoptimized wave front and for the optimized wave front [16]. Before optimization, the transmitted intensity forms a diffuse spot on the back surface of the sample. After optimization, a strong peak emerges in the target focus. The intensity increase in the center of the target was a factor of $746 \pm 28$. After optimization, $2.3 \%$ of the incident power is transmitted into the target focus.

More importantly, the intensity in an area with a radius of approximately $5 \mu \mathrm{m}$ around the target also increased, even though the algorithm did not use this intensity as feedback. This observation indicates that we have redistributed the incident light from closed eigenchannels to open eigenchannels. As a result of optimizing a single target point, the total angle-integrated transmission increased from 0.23 to 0.31 . This change amounts to a relative increase of $35 \%$.

For a quantitative analysis we need to know the degree of control $|\gamma|^{2}$. Factors like measurement noise and thermal drift result in a different degree of control for each single run of the experiment. Fortunately, it is possible to measure $|\gamma|^{2}$ directly for each run by observing the intensity in the target. Only the controlled fraction of the incident wave front contributes to the intensity in the target. The transmission to the target mode $\beta$ equals

$$
\begin{aligned}
\left|E_{\beta}^{\mathrm{act}}\right|^{2} & =\left|\sum_{a}^{N} t_{\beta a} E_{a}^{\mathrm{act}, \beta}\right|^{2} \\
& =|\gamma|^{2}\left|\sum_{a}^{N} t_{\beta a} E_{a}^{\mathrm{opt}, \beta}\right|^{2},
\end{aligned}
$$

where we used Eq. (4) and the fact that the error term is orthogonal to the ideal wave front. By substituting Eq. (2) we obtain

$$
\left|E_{\beta}^{\text {act }}\right|^{2}=\left|\gamma^{2}\right| T_{\beta}
$$

Equation (7) allows us to obtain the degree of control by measuring the intensity in the target focus $\left|E_{\beta}^{\text {act }}\right|^{2}$ and $T_{\beta}$. In the experimental procedure it is very impractical to measure $T_{\beta}$. Therefore, we approximate $T_{\beta}=T_{\text {tot }} N / M$. Here, $T_{\text {tot }}$ is the ensemble averaged total transmission of an unoptimized wave front, and $M$ is the number of transmitted free modes. Since our samples are sandwiched between a glass substrate on one side and air on the other side, the number of modes on the back of the sample is larger and $M=n^{2} N$, with $n=1.52$ the refractive index of the substrate. This approximation neglects the $C_{2}$ fluctuations [18] in the total transmission, which are in the order of $2 \%$ for our samples.

For the experimental run that is shown in Fig. 2, we find a degree of control of $|\gamma|^{2}=0.23$. This means that the incident field is a linear superposition of the perfectly shaped wave front (carrying $23 \%$ of the incident power) and a noise term (carrying the rest of the power). The total,

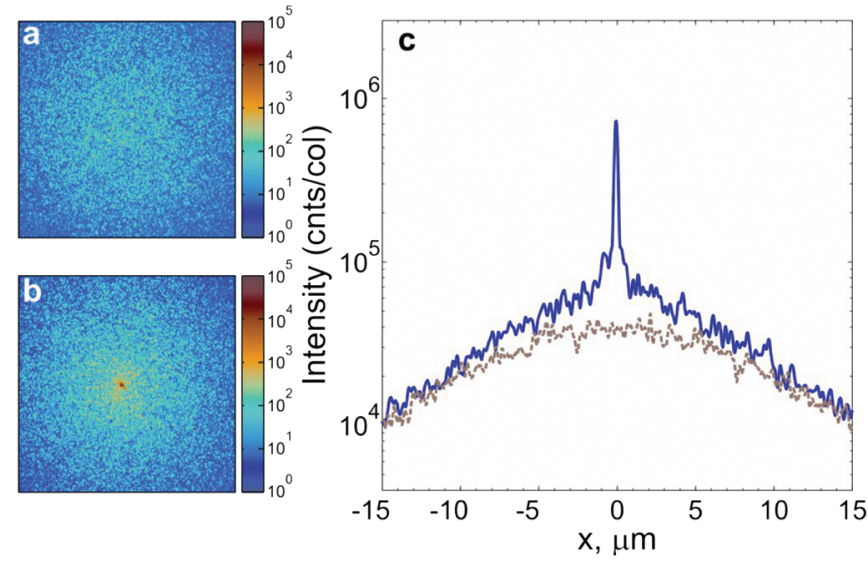

FIG. 2 (color). Intensity distribution of horizontally polarized light in a $30 \mu \mathrm{m} \times 30 \mu \mathrm{m}$ square area at the back of the sample. (a) For a nonoptimized incident wave front. (b) For an optimized wave front. (c) Intensity summed in the $y$ direction to average over speckle. Dashed curve, transmission of nonoptimized wave front; solid curve, transmission of optimized wave front.

angle-integrated transmission $T_{\text {tot }}^{\text {act }}$ contains contributions both from the perfectly shaped wave front and from the noise term,

$$
T_{\text {tot }}^{\text {act }}=T_{c}+\left(1-|\gamma|^{2}\right) T_{\text {tot }},
$$

where $T_{c}$ is the part of the transmission resulting from the perfectly shaped fraction of the incident wave front. By substituting Eq. (2) into Eq. (1) and summing the power in all transmitted free modes, we find

$$
T_{c}=|\gamma|^{2} \sum_{b}^{M} \frac{1}{T_{\beta}}\left|\sum_{a}^{N} t_{b a} t_{\beta a}^{*}\right|^{2} \equiv|\gamma|^{2} C_{4,2} .
$$

We evaluate $C_{4,2}$ theoretically by averaging over all possible target modes $\beta$. We assume that $C_{4,2}$ is selfaveraging, which is verified by our experiment. Neglecting small correlation terms between numerator and denominator we find

$$
C_{4,2}=\frac{1}{\left\langle T_{\beta}\right\rangle_{\beta}}\left\langle\sum_{b}^{M}\left|\sum_{a}^{N} t_{b a} t_{\beta a}^{*}\right|^{2}\right\rangle_{\beta}=\frac{\operatorname{Tr} t^{\dagger} t t^{\dagger} t}{\operatorname{Tr} t t^{\dagger}}
$$

From Eq. (10), it becomes clear that $C_{4,2}$ is a measure for the width of the distribution of the transmission eigenvalues [9]. By measuring the total transmission after optimizing the incident wave front, we have direct experimental access to this value for each single sample. Since we measured $|\gamma|^{2}$ separately, we can use Eqs. (8) and (9) to obtain $C_{4,2}$ from a single, nonideal experimental run. In the particular run in Fig. 2, we find $C_{4,2}=0.62$.

The ensemble averaged value for $C_{4,2}$ was derived using RMT. RMT [19] predicts $\left\langle C_{4,2}\right\rangle=2 / 3$ for a nonabsorbing system far away from the localization transition, regardless of the original transmission coefficient of the system. For a single realization of disorder, we found $C_{4,2}=0.62$. To 


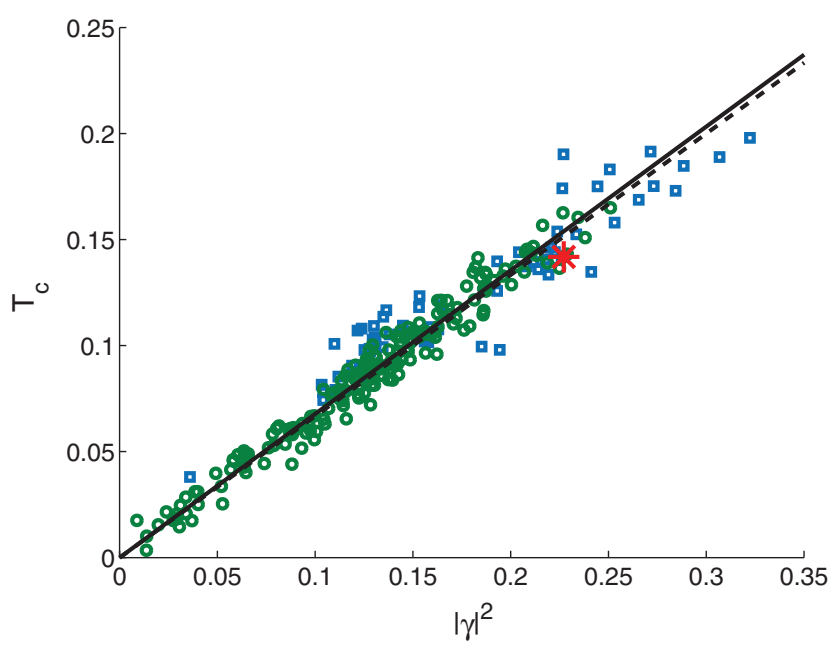

FIG. 3 (color). Total transmission of the controlled fraction of the wave front. Data from two different samples collapse to a universal curve. There are no adjustable parameters in the data processing or the theory. Circles, results for $11.3 \mu \mathrm{m}$-thick sample; squares, results for $5.7 \mu \mathrm{m}$-thick sample; star, data point corresponding to the experimental run that was shown in detail in Fig. 2; solid line, linear regression with a slope of 0.68 ; dotted line, slope of $2 / 3$ as predicted by RMT.

investigate the universality of this result and to compare the measured values with RMT, we performed automated sequences of measurements. The sample was translated between the runs to obtain different realizations of disorder.

The intensity increase was different for each of the optimizations in the sequence. The increase in the integrated transmission varied from a few percent to a maximum of $44 \%$. These variations are the result of drift due to varying environmental conditions. While drift is undesirable, it gives us a wide range of $|\gamma|^{2}$ to investigate. The degree of control $|\gamma|^{2}$ was determined for each of the runs using Eq. (7). Then, using Eq. (8), we isolated $T_{c}$.

In Fig. 3 we plotted $T_{\mathrm{c}}$ versus $|\gamma|^{2}$ for hundreds of measurements. To determine the effect of the sample thickness, two different samples were used: one with a thickness of $5.7 \pm 0.5 \mu \mathrm{m}$, and one that is approximately twice as thick $(11.3 \pm 0.5 \mu \mathrm{m})$. All data points collapse to a single line. Our data show that the transmission coefficient of an ideally shaped wave front does not depend on the sample thickness. A linear regression gives $\left\langle C_{4,2}\right\rangle=$ $0.68 \pm 0.07$ where the uncertainty follows from a worst case estimate of the systematical errors in the experiment. This value is in excellent agreement with RMT.

We have shown experimental evidence of controlled coupling of light into open transmission eigenchannels in opaque, strongly scattering materials. The coupling to open eigenchannels was enhanced by using a wave front shaping algorithm to optimize transmission to a focus, and detected by measuring the angle-averaged transmission intensity, which showed a relative increase of up to $44 \%$. We quantitatively compared the results for different samples and different realizations of disorder. All results showed a universal behavior that is in excellent quantitative agreement with random matrix theory. Our results demonstrate that RMT of wave transport can successfully be applied to open systems and single realizations of disorder. This conclusion is relevant for the propagation of electromagnetic waves, matter waves, and sound in open, strongly scattering environments.

We thank Ad Lagendijk and Willem Vos for valuable advice, and Elbert van Putten and Frerik van Beijnum for discussions. This work is part of the research program of the "Stichting voor Fundamenteel Onderzoek der Materie (FOM)," which is financially supported by the "Nederlandse Organisatie voor Wetenschappelijk Onderzoek" (NWO). A. P. Mosk is supported by a VIDI grant from NWO.

[1] M. P. van Albada and A. Lagendijk, Phys. Rev. Lett. 55, 2692 (1985)

[2] P. E. Wolf and G. Maret, Phys. Rev. Lett. 55, 2696 (1985).

[3] D. S. Wiersma, P. Bartolini, A. Lagendijk, and R. Righini, Nature (London) 390, 671 (1997).

[4] M. Störzer, P. Gross, C. M. Aegerter, and G. Maret, Phys. Rev. Lett. 96, 063904 (2006).

[5] T. Schwartz, G. Bartal, S. Fishman, and M. Segev, Nature (London) 446, 52 (2007).

[6] O. N. Dorokhov, Solid State Commun. 51, 381 (1984).

[7] Eigenchannels are specific to a single realization of disorder; they are a good basis to describe propagation of waves inside the sample. We use the term "free modes" for an orthogonal basis that describes free propagating waves outside the sample.

[8] Y. V. Nazarov, Phys. Rev. Lett. 73, 134 (1994).

[9] J.B. Pendry, A. MacKinnon, and A. B. Pretre, Physica (Amsterdam) 168A, 400 (1990).

[10] C. W. J. Beenakker, Rev. Mod. Phys. 69, 731 (1997).

[11] S. Washburn, IBM J. Res. Dev. 32, 335 (1988).

[12] J.F. de Boer, M.C. W. van Rossum, M.P. van Albada, T. M. Nieuwenhuizen, and A. Lagendijk, Phys. Rev. Lett. 73, 2567 (1994).

[13] F. Scheffold and G. Maret, Phys. Rev. Lett. 81, 5800 (1998).

[14] S. Hemmady, X. Zheng, E. Ott, T. M. Antonsen, and S. M. Anlage, Phys. Rev. Lett. 94, 014102 (2005).

[15] I. M. Vellekoop and A.P. Mosk, Opt. Lett. 32, 2309 (2007).

[16] See EPAPS Document No. E-PRLTAO-101-063834 for an appendix, specifying a detailed proof of the uniqueness of an optimal wave front as well as experimental details on the determination of an intensity reference. For more information on EPAPS, see http://www.aip.org/pubservs/ epaps.html.

[17] E. G. van Putten, I. M. Vellekoop, and A. P. Mosk, Appl. Opt. 47, 2076 (2008).

[18] R. Berkovits and S. Feng, Phys. Rep. 238, 135 (1994).

[19] P. A. Mello, P. Pereyra, and N. Kumar, Ann. Phys. (N.Y.) 181, 290 (1988). 